\title{
IDEAL TOPOLOGY ON A DISTRIBUTIVE LATTICE
}

\author{
P. V. RAMANA MURTY \\ (Received 15 November 1972; revised 16 August 1973) \\ Communicated by E. Strzelecki
}

\section{Introduction}

In [1] Atherton has asked whether the ideal topology (see [1]) is Hausdorff on every distributive lattice. He also gave some sufficient conditions (Kent's conditions $\mathrm{c} 1$ and $\mathrm{c} 3[1]$ ) under which the ideal topology on a complete distributive lattice is Hausdorff. In this paper we determine some more classes of lattices in which the ideal topology is Hausdorff and give examples to show that the classes determined are independent even in a complete distributive lattice. We also show by an example that Atherton's conditions are not necessary even in the case of a complete distributive lattice.

We prove that a distributive lattice with 0 and 1 is a Boolean algebra if and only if the set theoretic complement of every ultrafilter is a maximal ideal (see theorem 1). We also prove that the ideal topology is Hausdroff on any lattice in which either every completely irreducible ideal is principal or every completely irreducible filter is principal (see theorem 7). Further we show that the ideal topology is Hausdorff on every chain and on any distributive lattice which is either disjunctive and atomistic or dual disjunctive and dual atomistic (see theorems 6 and 8 ). We also observe that the ideal topology is $T_{1}$ on any lattice (see theorem 3).

It is well known that in a Boolean algebra the set theoretic complement of a maximal ideal is an ultrafilter and vice versa. We show now in theorem 1 that this is a characteristic property of the Boolean algebra by proving that a distributive lattice with 0 and 1 is a Boolean algebra if and only if the complement of every maximal ideal is an ultrafilter.

THEOREM 1. Let $L$ be a distributive lattice with 0 and 1 . Then the following statements are equivalent.

(i) L is a Boolean algebra.

(ii) Complement of every ultrafilter in $L$ is a maximal ideal.

(iii) Complement of every maximal ideal is an ultrafilter. 
(iv) Every prime filter is an ultrafilter.

(v) Every prime ideal is a maximal ideal.

Proof.(i) $\Rightarrow$ (ii), (iii), (iv), (v) are all obvious. Assume (ii). Let $x \in L$ and let $T(x)=\{y \in L \mid x \cap y=0\}$ and $D(x)=\{y \in L \mid x \cup y=1\}$. If $T(x) \cap D(x)$ $\neq \varnothing$, then we are through. Suppose $T(x) \cap D(x)=\varnothing$. It is easy to observe that $T(x)$ is an ideal and $D(x)$ is a dual ideal of $L$. Now consider the family $\mathscr{P}$ of all dual ideals $F$ such that $F \supseteq D(x)$ and $F \cap T(x)=\varnothing$. Clearly $D(x) \in \mathscr{S}$. Applying Zorn's lemma we have a maximal element $F$ of $\mathscr{S}$. If $x \notin F$ then the filter generated by $F$ and $x$ intersects $T(x)$. Hence there is an element $y \in T(x)$ such that $y \geqq f \cap x$ for some $f \in F$. Now $0=y \cap x \geqq f \cap x$ so that $f \in T(x)$ which is impossible since $F \cap T(x)=\varnothing$. Hence $x \in F$. We now show that $F$ is an ultrafilter. Let $z \notin F$. Then the filter generated by $F$ and $z$ intersects $T(x)$. Hence there is an element $y \in T(x)$ such that $y \geqq f \cap z$ for some $f \in F$. Now $0=y \cap x \geqq f \cap z \cap x$. Since $x \in F$ and $f \in F$ we have $f \cap x \in F$ and $z \cap f \cap x=0$. Hence $F$ is an ultrafilter so that by assumption $L-F$ is a maximal ideal and $x \notin L-F$. Hence there exists an element $y \in L-F$ such that $x \cup y=1$ which is impossible since $D(x) \subseteq F$. Hence $T(x) \cap D(x) \neq \varnothing$ and (ii) $\Rightarrow$ (i). (iii) $\Rightarrow$ (i) follows by duality. Assume (iv). Let $M$ be a maximal ideal of $L$ so that $L-M$ is a prime filter of $L$ and hence by assumption $L-M$ is an ultrafilter. Thus (iv) $\Rightarrow$ (iii) and similarly $(\mathrm{v}) \Rightarrow$ (ii).

In a Boolean algebra maximal ideals and completely irreducible ideals (see [1]) coincide and ultrafilters and completely irreducible filters coincide. Hence one may ask whether the words ultra and maximal in (ii) may be replaced by completely irreducible filter and completely irreducible ideal. We answer in the negative by giving the following

EXAmPLE 1. Let $L$ be the set of all non-negative integers under divisibility ordering (i.e., $a \leqq b$ if and only if $a$ divides $b$ ). Then it is well known that $L$ is a distributive lattice with integer 1 as the least element and integer 0 as the greatest element. It can be shown that every completely irreducible ideal is of the form $\left\{x \mid p^{\alpha} \nmid x\right\}$ for some prime power $p$ and every completely irreducible filter is of the form $\left\{x\left|p^{\alpha}\right| x\right\}$ and yet $L$ is not a Boolean algebra.

In a distributive lattice every completely irreducible ideal is prime and every completely irreducible filter is prime. Hence one may try to replace the word prime filter in (iv) by completely irreducible filter. We show that it is impossible by giving the following

Example 2. Let $L$ be the set of all finite subsets of an infinite set $N$ together with $N$. Then $L$ is a distributive lattice with 0 and 1 under the usual set inclusion. In this lattice it can be easily seen that every completely irreducible filter is ultra and yet $L$ is not a Boolean algebra. 
THEOREM 2. Let $L$ be any lattice. Then every proper ideal of $L$ is the intersection of all completely irreducible ideals that contain it and dually every proper filter is the intersection of all completely irreducible filters that contain it.

Proof: If $x \in L-I_{0}$, where $I_{0}$ is the given ideal, we obtain by Zorn's lemma an ideal $I_{x} \supseteq I_{0}$ which is maximal subject to not containing $x$. Clearly $I_{x}$ is completely irreducible and $I_{0}=\bigcap\left\{I_{x} \mid x \in L-I_{0}\right\}$, from which the theorem follows.

Theorem 3. Let $L$ be a lattice. Then the ideal topology on $L$ is $T_{1}$.

Proof. Let $x \in L$ and let $x \neq y$ so that either $x \leq y$ or $y \leq x$. Suppose $x \$ y$. Since the ideal $\{a \mid a \leqq y\}$ does not contain $x$ there exists by Theorem 2 a completely irreducible ideal (hence an open set) containing $y$ and not containing $x$. By a dual argument in the case $y \leq x$ we get an open set containing $y$ and not containing $x$ so that the ideal topology on $L$ is $T_{1}$.

COROLlaRY 1. The ideal topology is discrete on any finite lattice.

From now onwards we suppose that $L$ is a lattice endowed with the ideal topology.

THEOREM 4. The mappings $(x, y) \rightarrow(x \cup y$ and $(x, y) \rightarrow x \cap y$ from $L \times L \rightarrow L$ are continuous if $L$ is distributive.

Proof. Let $f: L \times L \rightarrow L$ be defined by $f(x, y)=x \cup y$. Let $x \cup y \in G$ where $G$ is a subbasic neighbourhood of $x \cup y$. Suppose $G$ is a completely irreducible ideal. Then both $x$ and $y$ belong to $G$ and $f(G \times G) \subseteq G$. If $G$ is a completely irreducible dual ideal then either $x$ or $y \in G$ (since $G$ is a prime dual ideal). If $x \in G$, then $G \times L$ is a neighbourhood of $(x, y)$ and $f(G \times L) \subseteq G$. Thus $f$ is continuous. By a dual argument it can be shown that $(x, y) \rightarrow$ $x \cap y$ is also continuous.

The above theorem is true if $L$ is a finite lattice because of corollary 1 .

REMARK 1. It can be easily observed that principal ideals and principal dual ideals of $L$ are closed in $L$ (see page 258 under corollary 3 in [3]).

THEOREM 5. If $L_{1}, \cdots, L_{n}$ are lattices in each of which the ideal topology is Hausdorff, then the ideal topology on the product lattice $L_{1} \times L_{2} \times \cdots \times L_{n}$ is Hausdorff.

Proof. It is easy to see that the product topology on the product lattice is coarser than the ideal topology on it. Since the product topology is Hausdorff it follows that the ideal topology on $L_{1} \times \cdots \times L_{n}$ is Hausdorff.

We shall now determine a few classes of distributive lattices in which the ideal topology is Hausdorff.

THEOREM 6. If $L$ is a chain, then the ideal topology on $L$ is Hausdorff. 
Proof. It can be easily observed that for each $a$ in $L, I_{a}=\{x \in L \mid x<a\}$ and $D_{a}=\{x \mid x>a\}$ are respectively completely irreducible ideal and completely irreducible dual ideals. Let $a, b \in L$ and $a<b$. Suppose there is an $r \in L$ such that $a<r<b$. Then $I_{r}$ and $D_{r}$ are respectively neighbourhoods of $a$ and $b$ with $I_{r} \cap D_{r}=\varnothing$. If there is no $r$ in $L$ such that $a<r<b$, then $I_{b}$ and $D_{a}$ are respectively neighbourhoods of $a$ and $b$ with $I_{b} \cap D_{a}=\varnothing$. Thus $L$ is Hausdorff.

The following theorem is valid on any lattice i.e., the assumption that $L$ is distributive is not made.

THEOREM 7. Let L be a lattice endowed with the ideal topology. If either every completely irreducible ideal is principal or every completely irreducible filter is principal, then the ideal topology on L is Hausdorff.

Proof. Let $x, y \in L$ and let $x \neq y$ so that either $x \neq y$ or $y \leq x$. If $x \neq y$, then there exists a completely irreducible ideal $I$ containing $y$ and not containing $x$. Now since $I$ is principal (by hypothesis) by Remark 1 we have $I$ is closed so that $L-I$ is an open set containing $x$ and $I \cap(L-I)=\varnothing$. Similarly in case $y$ we can obtain disjoint neighbourhoods for $x$ and $y$. Thus $L$ is a Hausdorff space. By a dual argument we can show that $L$ is Hausdorff in case every completely irreducible dual ideal is principal.

Definition 1. A lattice $L$ with 0 is called disjunctive if $x, y \in L$ and $x<y$ implies that there exists an element $c$ in Lwith $x \cap c=0$ and $y \cap c \neq 0$. Dually a lattice with 1 is called dual disjunctive if $x, y \in L$ and $x>y$ implies the existence of an element $c$ in $L$ with $x \cup c=1$ and $y \cup c \neq 1$ (see Birkhoff [2], page 173).

Lemma 1. A lattice $L$ with 0 is atomistic and disjunctive if and only if given $x, y \in L$ with $x<y$ there exists an atom $p \leqq y$ such that $p \cap x=0$.

Proof. If the condition is satisfied then obviously $L$ is atomistic and the atom $p$ plays the role of $c$. Conversely suppose that $L$ is atomistic and disjunctive and let $x<y$. There is an element $c$ in $L$ with $x \cap c=0$ and $y \cap c \neq 0$ so that we have an atom $p \leqq y \cap c$. Then $p \cap x \leqq y \cap c \cap x \leqq c \cap x=0$ and $p \leqq y$.

THEOREM 8. Let $L$ be a distributive lattice with 0 and 1 . If either $L$ is atomistic and disjunctive or dual atomistic and dual disjunctive, then the ideal topology on L is Hausdorff.

Proof. Let $x, y \in L$ with $x \neq y$ so that either $x \leq y$ or $y \leq x$. If $x y$, then $y<x \cup y$ so that by Lemma 1 there is an atom $p \leqq x \cup y$ with $p \cap y=0$. Hence $p=p \cap(x \cup y)=(p \cap x) \cup(p \cap y)=p \cap x$ so that $p \leqq x$. It can be easily observed that $I=\{z \in L \mid p \cap z=0\}$ is an ideal maximal with respect to the property of not containing $p$ so that $I$ is a completely irreducible ideal 
containing $y$ and not containing $x$. The dual ideal $J$ generated by $p$ being an ultrafilter is a completely irreducible dual ideal containing $x$ and not containing $y$. Thus $I$ is an open set containing $y$ and $J$ is an open set containing $x$ with $I \cap J=\varnothing$. Hence the ideal topology on $L$ is Hausdorff. By a dual argument it follows that $L$ is Hausdorff if it is dual atomistic and dual disjunctive.

We now give examples to show that the classes determined are independent even in the case of a complete distributive lattice.

EXAMPLE 1 . The closed interval $[0,1]$ of the real number system is a complete distributive lattice under the natural ordering. It can be easily seen that it is neither atomistic nor dual atomistic. It can also be observed that every completely irreducible ideal is non-principal and every completely irreducible filter is nonprincipal.

REMARK 2. The above example also shows that Atherton's conditions [1] for the ideal topology on a complete distributive lattice to be Hausdorff are not necessary.

EXAmple 2. Let $L$ be the same lattice as in example 1 of Theorem 1. It can be easily observed that every dual ideal is principal and the set of all odd integers is a completely irreducible ideal which is not principal. It can also be observed that $L$ is neither disjunctive nor dual atomistic. Finally it is obvious that $L$ is not a chain.

EXAMPLE 3. Let $L$ be the set of all countable subsets of the real line $\mathbb{R}$ together with $\mathbb{R}$ under the usual set inclusion. This lattice is a disjunctive and atomistic distributive lattice. Also $\{X \in L \mid \mathbb{Z}-X$ is a finite subset of $\mathbb{R}, \mathbb{Z}$ being the set of integers $\}$ is a proper filter contained in an ultrafilter which is non-principal and $L-\{R\}$ is a non-principal maximal ideal. Clearly $L$ is neither dual atomistic nor a chain.

DEFINITION 2. Let $L$ be a distributive lattice. The prime ideal topology on Lis the topology on Lhaving an open subbase consisting of all prime ideals and prime filters of $L$.

REMARK 3. In $\$ 1$ of [1] Atherton proves some results about the ideal topology on a Boolean algebra. All these results depend on the fact that in a Boolean algebra maximal ideals and completely irreducible ideals coincide and dually ultrafilters and completely irreducible filters coincide, we note that if we assume that $L$ is merely distributive and consider the prime ideal topology the same results can be proved since the complement of a prime ideal is a prime filter and vice versa. The proofs are identical with Atherton's needing only the fact that in a distributive lattice completely irreducible ideal is prime and dually completely irreducible filter is prime. Theorem 5 of [3] (the ideal topology on a Boolean algebra is Hausdorff) follows as a corollary. 
In conclusion I thank Professor Dr. N. V. Subrahmanyam for his valuable . comments and suggestions during the preparation of this revised paper. I also thank the referee whose valuable comments helped in shaping the paper into its present form.

\section{References}

[1] C. R. Atherton, 'Concerning intrinsic topologies on Boolean algebras and certain bicompactly generated lattices', Glasgow Mathematical Journal 11, (1970), 156-161.

[2] G. Birkhoff, 'Lattice theory', Amer. Math. Colloquium publications (25), (1948).

[3] A. J. Ward, 'On relations between certain intrinsic topologies in partially ordered sets', Proc. Cambridge Philos. Soc. 51 (1955), 254-261.

Department of Mathematics

Andhra University

Waltair - 530003, India 